\title{
Data mining of the expression and regulatory role of BCAT1 in hepatocellular carcinoma
}

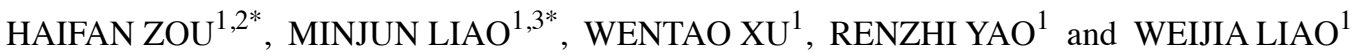 \\ ${ }^{1}$ Laboratory of Hepatobiliary and Pancreatic Surgery, Affiliated Hospital of Guilin Medical University; \\ ${ }^{2}$ Scientific Experiment Center, Guilin Medical University, Guilin, Guangxi 541001; ${ }^{3}$ Clinical School \\ of Medicine, Guangxi Medical University, Nanning, Guangxi 530021, P.R. China
}

Received May 10, 2019; Accepted September 6, 2019

DOI: $10.3892 / \mathrm{ol} .2019 .10932$

\begin{abstract}
Branched chain amino acid transaminase 1 (BCAT1) catalyzes the production of glutamates and branched-chain $\alpha$-ketoacids from branched chain amino acids, and a normal BCAT1 expression is associated with tumorigenesis. Sequencing data from public databases, including The Cancer Genome Atlas, was used to analyze BCAT1 expression and regulation networks for hepatocellular carcinoma (HCC). Expression and methylation were assessed using UALCAN analysis, and data from multiple datasets concerning the $B C A T 1$ expression level and associated survival rates were further analyzed using HCCDB; interaction networks of biological function were constructed using GeneMANIA. LinkedOmics was used to indicate correlations between $B C A T 1$ and any identified differentially expressed genes. Gene enrichment analysis of $B C A T$-associated genes was conducted using the Web-based Gene SeT AnaLysis Toolkit. The expression levels of $B C A T 1$ were increased in patients with HCC and in most cases, the level of $B C A T 1$ promoter methylation was reduced. Interaction network analysis suggested that $B C A T 1$ was involved in 'metabolism', 'carcinogenesis' and the 'immune response' via numerous cancer-associated pathways. The present study revealed the expression patterns and potential function networks of $B C A T 1$ in $\mathrm{HCC}$, providing insights for future research into the role of $B C A T 1$ in hepatocarcinogenesis. In addition, the study provided researchers with a way to analyze the genes of interest so they can continue their research in the right direction.
\end{abstract}

Correspondence to: Professor Weijia Liao or Dr Renzhi Yao, Laboratory of Hepatobiliary and Pancreatic Surgery, Affiliated Hospital of Guilin Medical University, 15 Lequn Road, Guilin, Guangxi 541001, P.R. China

E-mail: liaoweijia288@163.com

E-mail: yaorenzhi_gzb@163.com

*Contributed equally

Key words: branched chain amino acid transaminase 1, hepatocellular carcinoma, expression, network analysis, data mining

\section{Introduction}

With the sixth highest incidence and fourth highest mortality rates, liver cancer is one of the most important causes of cancer-associated mortality worldwide. In 2018, 840,000 cases of liver cancer were diagnosed in addition to 782,000 mortalities, of which $\geq 85 \%$ were associated with hepatocellular carcinoma (HCC) $(1,2)$. Although the development of predictive and treatment methods has increased the survival rates of patients with HCC, the early diagnosis and 5-year survival rates remain poor (2). Clinically, HCC can be diagnosed or prognosed using ultrasound, computerized tomography and the detection of serum $\alpha$-fetoprotein (AFP) levels (3). However, the sensitivity and specificity of these methods is unsatisfactory. The complexity of the molecular mechanisms of HCC has prevented the elucidation of its development and therefore, the means with which to successfully treat the disease. Thus, it is necessary to identify novel therapeutics and drug targets by screening HCC-associated gene networks.

Branched chain amino acid transaminase 1 (BCAT1) is able to catalyze the synthesis of $\alpha$-ketoglutarates from branched chain amino acids (BCAAs) and the subsequent production of glutamates and branched-chain $\alpha$-ketoacids. BCAT1 is reportedly involved in the progression of various malignancies, including breast (4), ovarian (5), gastric (6) and pancreatic cancer $(7)$, as well as HCC $(8,9)$. The overexpression of BCAT1 promoted cellular proliferation, migration and invasion in various types of tumor via multiple signaling pathways $(10,11)$. In addition, previous studies have revealed that $B C A T 1$ is involved in DNA methylation and associated with inflammatory diseases $(12,13)$. However, one study has suggested that BCAT1 expression is not associated with the overall survival of patients with HCC (14), thus its exact role and mechanism in hepatocarcinogenesis remain controversial.

In a previous study, $B C A T 1$ expression levels were upregulated in the corresponding HCC tissues. Thus, to further investigate the role of $B C A T 1$ in $\mathrm{HCC}$, patient data from The Cancer Genome Atlas (TCGA) database and Gene Expression Omnibus (GEO) datasets (https://www.ncbi. nlm.nih.gov/gds) was used to assess the expression levels of $B C A T 1$ in patients with HCC. Functional networks of $B C A T 1$ in HCC were also analyzed. The results revealed that $B C A T 1$ 
may represent a novel therapeutic and drug target for HCC, which may provide insights for future research and the development of treatment strategies.

\section{Materials and methods}

UALCAN analysis. UALCAN (http://ualcan.path.uab.edu) is a publicly available interactive online portal used to perform in-depth analyses of TCGA gene expression data (15). UALCAN can be used to determine methylation and relative expression levels, as well as the survival of a target gene across several clinicopathological features. Expression levels were measured as transcripts per million. Promoter DNA methylation information was from the TCGA Infinium Human Methylation 450K BeadChip arrays. The $t$ test was performed using a PERL script with Comprehensive Perl Archive Network module (http://search. cpan.org/ yunfang/Statistics-TTest-1.1.0/TTest.pm) to compare the statistical significance between two independent groups.

GeneMANIA analysis. GeneMANIA (http://genemania.org/) is a web interface that uses large sets of functional association data to identify single genes related to a set of input genes (16). Association data include protein and genetic interaction pathways, co-expression, co-localization and protein domain homology. GeneMANIA was used to construct the BCATI biological network.

LinkedOmics analysis. The LinkedOmics database (http://www.linkedomics.org/login.php) contains the multi-omics and clinical data of 32 cancer types, and a total of 11,158 patients from TCGA project (17). LinkFinder, one of three modules, was used to identify differentially expressed genes (DEGs) in TCGA LIHC cohort whose expression levels correlated with those of $B C A T 1$. The searching and targeting datasets were both of 'RNAseq', and the results were statistically analyzed using Pearson's correlation coefficient. LinkInterpreter, a module that recruited Web-based Gene SeT AnaLysis Toolkit, was used to determine the pathways and networks in which the identified DEGs were involved (18).

HCCDB analysis. The HCCDB database (http://lifeome. net/database/hccdb/home.html) comprises $\sim 4,000$ clinical samples from 15 public HCC expression datasets (13 GEO microarray datasets and two RNA-Seq datasets, TCGALICH and ICGC LIRI-JP) and serves as a one-stop online resource for the investigation of HCC gene expression (19). All GEO datasets are available from the GEO repository (https://www.ncbi.nlm.nih.gov/gds). The accession numbers are: GSE25097 (20-23), GSE22058 (24,25), GSE36376 (26), GSE14520 (27-30), GSE10143 (31), GSE9843 (32-35), GSE19977 (33,34), GSE46444 (36), GSE54236 (37,38), GSE63898 (39), GSE43619 (40), GSE64041 (41) and GSE76427 (42). ICGC LIRI-JP is available on the IGCG Data Portal (https://dcc.icgc.org/projects/LIRI-JP) and TCGA-LIHC is available on TCGA (https://portal.gdc. cancer.gov/projects/TCGA-LIHC). HCCDB was used to analyze the $B C A T 1$ co-expression networks of HCC and the corresponding adjacent tissues. The expression levels of BCATl in different datasets were also detected. Samples were classified into high-/low-expression groups by median expression value of $B C A T 1$.

\section{Results}

Expression and promoter methylation levels of BCAT1 in $H C C$ tissues. The expression levels of BCATI in HCC tissues were investigated using a number of datasets from TCGA. The data revealed that the mRNA expression levels of $B C A T 1$ were significantly increased in HCC tissues compared with normal tissues $(\mathrm{P}<0.001$; Fig. 1). Combined with the authors' previous results (43), $B C A T 1$ may serve as a novel diagnostic marker of HCC. Secondly, the level of BCAT1 promoter methylation in HCC tissues was also assessed. The data suggested the same trend as that of BCAT1 mRNA expression (Fig. 2); the higher the mRNA expression level, the lower the methylation level. Subsequently, data from 12 additional GEO datasets were analyzed (12 of the 15 datasets in the HCCDB database; three were not included because analysis results were not returned by HCCDB tools), which revealed contradictions between the results of different studies (Fig. 3). Studies including dataset 4, 6 and 8 revealed no significant differences in the mRNA expression levels of $B C A T 1$ between $\mathrm{HCC}$ and adjacent normal tissues.

Effects of BCATl on the survival of patients with HCC. In tumors, the abnormal expression of specific genes has long been associated with overall patient survival. In the present study, two datasets from HCCDB were used to analyze the overall survival of HCC patients with high and low BCATI expression levels. This revealed that where the expression level of $B C A T 1$ was not significantly different between the HCC and adjacent tissues, the overall survival of patients in the high- and low-BCATl expression cohorts did not differ significantly (Fig. 4A). By contrast, if the expression level of $B C A T 1$ in HCC tissues was significantly increased compared within the adjacent tissues, patients with high $B C A T 1$ expression levels possessed a lower overall survival probability than those with low expression levels (Fig. 4B).

Interaction network of BCAT1. A BCATl interaction network was constructed to identify potential interactions between BCAT1and other cancer-associated proteins. The results showed that BCAT1 could be co-expressed with phosphoserine aminotransferase, was predicted to interact with 13 proteins, shared protein domains with its isoform BCAT2 and directly interacted with six proteins (Fig. 5). These proteins are involved in 'metabolic pathway', 'amino acids biosynthesis and degradation', 'myc pathway', 'p53 signaling pathway' and 'other cancer-related pathways or biological processes'.

LinkedOmics online tools were then used to investigate the genes that were co-expressed with BCAT1 in HCC. A volcano plot revealed that the expression of 2,970 genes negatively correlated with that of $B C A T 1$ [green spot; false discovery rate (FDR) <0.05], while the expression of 9,077 genes positively correlated with BCTAl (red spot; FDR <0.05; Fig. 6A). The top 50 negatively and positively correlated genes are displayed in Fig. $6 \mathrm{~B}$ and $\mathrm{C}$. These results indicate that $B C A T 1$ serves a critical role in HCC development. Pearson's correlation coefficient analysis revealed a strong positive association between 
the expression levels of BCAT1 and plasminogen activator, urokinase receptor (Pearson's correlation coefficient $=0.7142$; $\mathrm{P}=3.89 \times 10^{-59}$; Fig. 7A), RELT (Pearson's correlation coefficient $=0.6708 ; \mathrm{P}=7.628 \times 10^{-50}$; Fig. $\left.7 \mathrm{~B}\right), F_{c}$ fragment of $I g G$ receptor $I c$ (Pearson's correlation coefficient $=0.6651$; $\mathrm{P}=9.94 \times 10^{-49}$; Fig. 7C), and a negative association with $M L X$ interacting protein like (MLXIPL; Pearson's correlation coefficient $=-0.5685 ; \mathrm{P}=3.787 \times 10^{-33}$; Fig. 7D), RAR related orphan receptor $C(R O R C$; Pearson's correlation coefficient $=-0.5677$; $\mathrm{P}=4.868 \times 10^{-33}$; Fig. 7E) and pyruvate dehydrogenase kinase 2 (PDK2; Pearson's correlation coefficient $=-0.5421$; $\mathrm{P}=1.001 \times 10^{-29}$; Fig. $7 \mathrm{~F}$ ), which are involved in 'extracellular matrix degradation', 'NF- $\mathrm{B}$ pathway and immune response', 'IgG component', 'triglyceride synthesis', 'lymphoid organogenesis and thymopoiesis' and 'pyruvate dehydrogenation', respectively.

Biological process and molecular function analyses were conducted using gene set enrichment analysis, which showed that $B C A T$ 1-associated DEGs were involved in a number of biological processes and molecular functions, including 'protein activation cascade', 'respiratory burst', 'T cell activation', 'sphingolipid binding', 'MHC protein binding' and 'cytokine receptor activity' (Fig. 8). These data indicate that $B C A T 1$ serves an important role in immune system activation, cellular responses to stimulation, metabolism and a number of other processes.

\section{Discussion}

The proliferation of cancer cells depends on extra nutrients acquired from the tumor microenvironment $(44,45)$. BCAAs are essential for tumor growth and the progression of multiple biological pathways (46). A number of studies have revealed that the enzymes catalyzing BCAAs into their corresponding nutrients are predominantly overexpressed in cancer $(7,13,47)$ and that BCAT1 is one of the most important enzymes involved in BCAA catabolism. The authors' previous study indicated that $B C A T 1$ was significantly upregulated in $\mathrm{HCC}$ and that this was associated with poor patient prognosis (43). To further investigate the function networks of $B C A T 1$ in $\mathrm{HCC}$, bioinformatics analyses were performed using a selection of public databases to provide insights for future research into the metabolic regulation of HCC.

With a lack of appropriate markers of early stage HCC, $70-80 \%$ of patients are diagnosed at the advanced stage of disease and lose the opportunity to undergo surgery. Clinically, AFP has long been regarded as the standard for liver cancer diagnosis; however, a large number of $\mathrm{HCC}$ patients are AFP-negative (2), thus additional biomarkers for the early detection of HCC are required. A previous study explored the use of serum methylated $B C A T 1$ and IKAROS family zinc finger 1 (IKZF1) for the detection of colorectal neoplasia; this revealed that for the diagnosis of colorectal cancer, the detection of BCAT1/IKZF1 DNA in the serum had a comparable sensitivity, but a higher specificity than the traditional fecal immunochemical test (48). The present study demonstrated that BCAT1 mRNA expression levels in HCC tissues were increased compared with those in normal tissues. In addition, the level of $B C A T 1$ promoter methylation was decreased in HCC tissues compared within

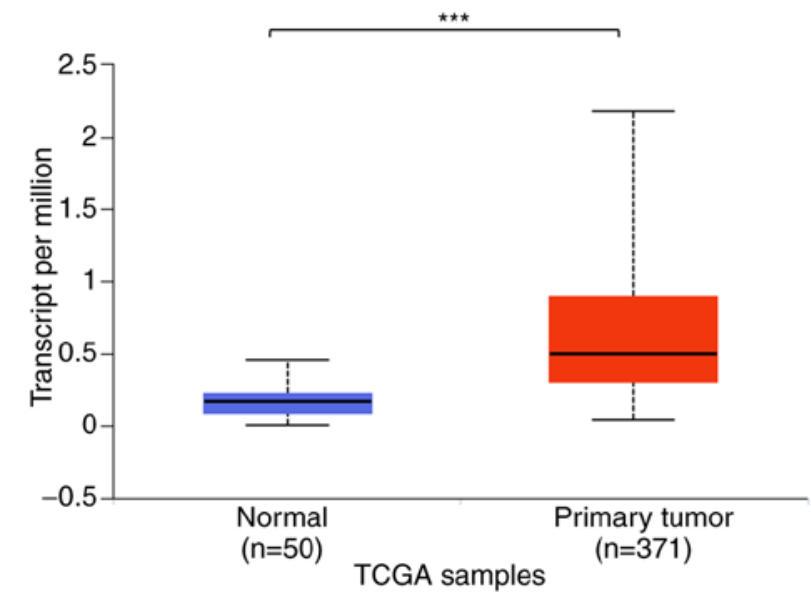

Figure 1. BCAT1 expression level in $\mathrm{HCC}$ and different classifications. Relative expression of $B C A T 1$ in normal and HCC tissues. Data are presented as the mean \pm standard error. ${ }^{* * *} \mathrm{P}<0.001$. Transcripts per million was used to measure the expression. TCGA, the Cancer Genome Atlas; BCAT1, branched chain amino acid transaminase 1 ; HCC, hepatocellular carcinoma.

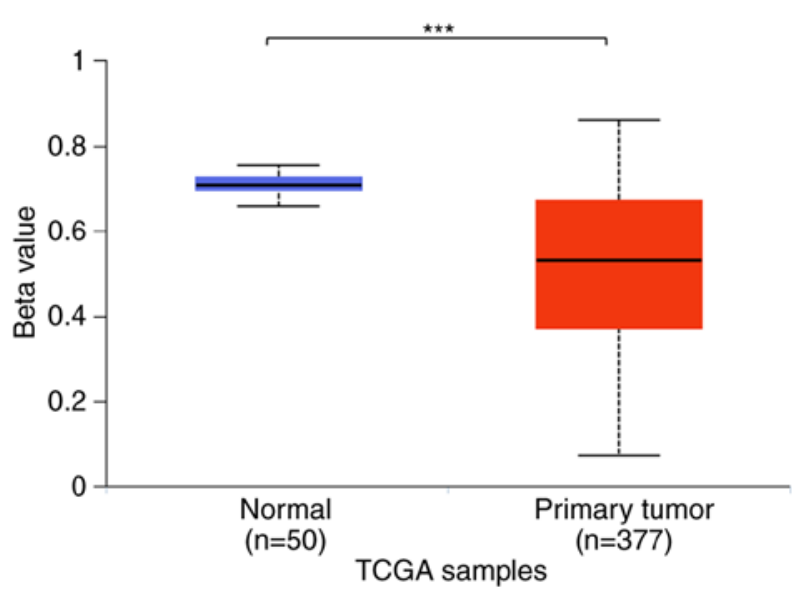

Figure 2. Promoter methylation level of $B C A T 1$ in HCC. Methylation level of $B C A T 1$ in normal and HCC tissues. Data are presented as the mean \pm standard error. ${ }^{* * *} \mathrm{P}<0.001$. BCAT1, branched chain amino acid transaminase 1 ; HCC, hepatocellular carcinoma; TCGA, The Cancer Genome Atlas.

normal tissues as that indicated for mRNA expression levels. However, analysis of 12 datasets from different regions indicated that in some cases, the expression level of BCAT1 was not significantly different between the HCC and adjacent normal tissues. These results suggested that while BCAT1 may serve as a marker for the detection of HCC, regional variations may apply. For this reason, further large-scale, multicenter studies are required to confirm the role of BCATI in the prediction and diagnosis of HCC. Nonetheless, the analysis of $B C A T 1$ expression and promoter methylation levels inpatients demonstrated its potential use as a reliable biomarker of HCC.

The abnormal expression of oncogenes is closely associated with patient survival $(49,50)$. Overexpression of MTHFD1 predicted poorer overall survival in patients with HCC (51). Also, the expression levels of SIRT1weresignificantlyincreasedin HCC and associated with poor patient survival (52). In this study, although the 


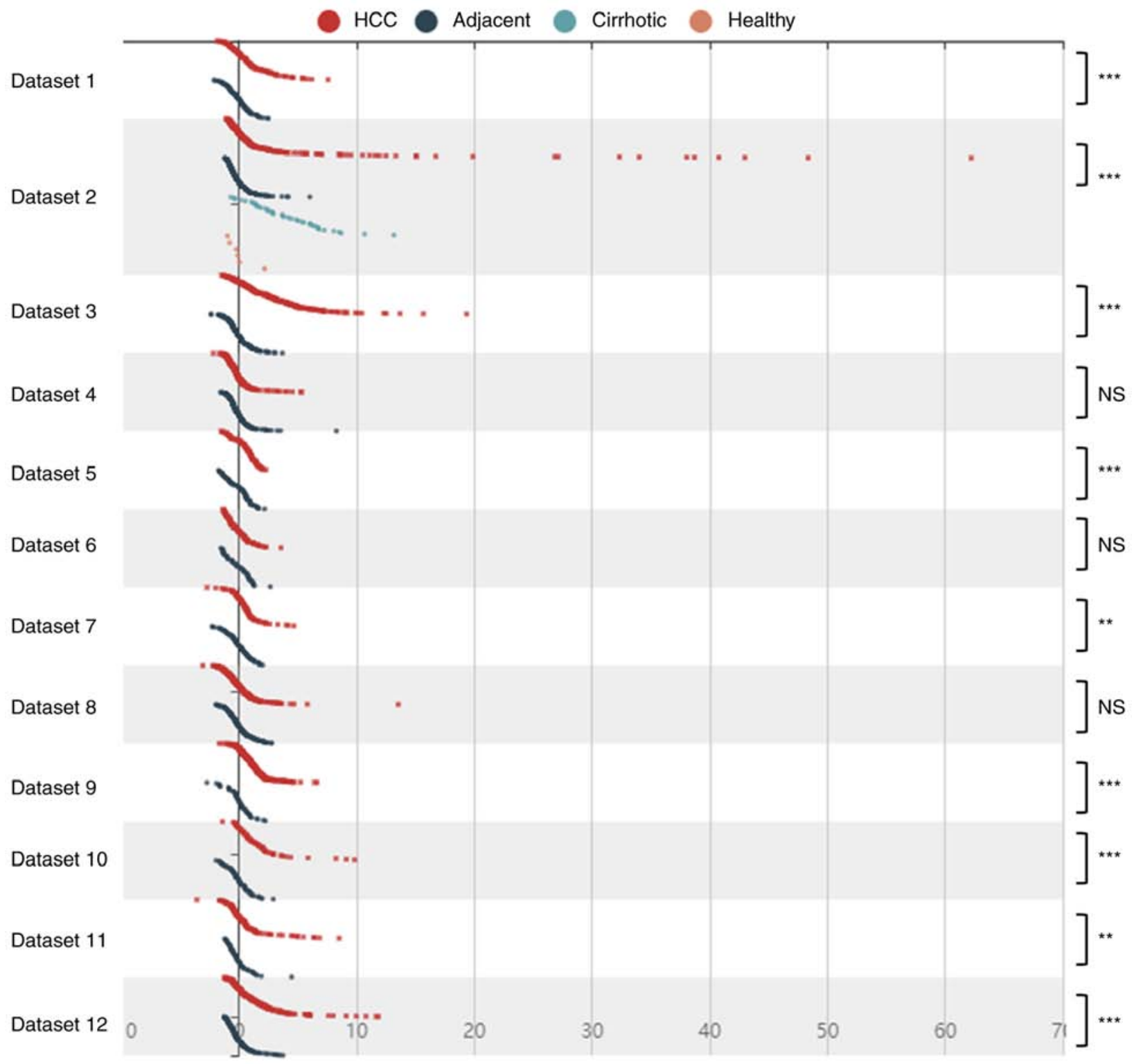

Scaled expression

Figure 3. Expression levels of $B C A T 1$ in different datasets. A total of 9 of 12 datasets showed that expression levels of $B C A T 1$ in $\mathrm{HCC}$ tissues are significantly increased compared with in adjacent tissues, but 3 of 12 datasets showed no significant difference between the expression levels of $B C A T 1$ in HCC tissues and in adjacent tissues. ${ }^{* *} \mathrm{P}<0.01$ and ${ }^{* * *} \mathrm{P}<0.001$. BCAT1, branched chain amino acid transaminase 1 ; HCC, hepatocellular carcinoma; NS, not significant.

majority of studies illustrated an upregulation in BCAT1 expression levels in HCC tissues (compared with adjacent tissues), selected cases presented an opposing result. Notably, further analyses within the present study indicated that patients with no significant differences in the level of $B C A T 1$ expression between tumor and paracancerous tissues possessed a $B C A T 1$ expression-independent survival rate. Meanwhile, patients with higher $B C A T 1$ expression levels in HCC tissues exhibited a $B C A T 1$-dependent survival probability. These results suggest that additional methods may be required to demonstrate the role of BCAT1 in HCC; this may include not only focusing on mRNA and protein expression, but also assessing the products of $B C A T 1$ enzymatic reactions. BCAT1 is one of the key enzymes involved in amino acid metabolism, such that a repeatable method such as metabolomics analysis may further clarify the role of
$B C A T 1$. However, the results ultimately revealed that $B C A T 1$ serves an important role in the survival of patients with HCC.

Tumorigenesis is a complex process with abnormal changes in protein-protein interactions and signaling pathways (53). BCAT1 was identified to be involved in the proliferation $(4,54)$, apoptosis $(55)$, invasion and metastasis (56) of cancer cells, and to be associated with poor prognosis inpatients with various types of cancer $(57,58)$. In addition, $B C A T 1$ was reported to regulate mTOR signaling (59), as well as the Wnt (60) and FoxO signaling pathways (61). However, the effects of BCAT1 on other pathways and cellular functions remain unclear.

In the present study, the expression of $B C A T 1$-associated genes was investigated and $B C A T 1$ was found to be involved in various other signaling pathways and biological processes; to the best of our knowledge, the relationship between 

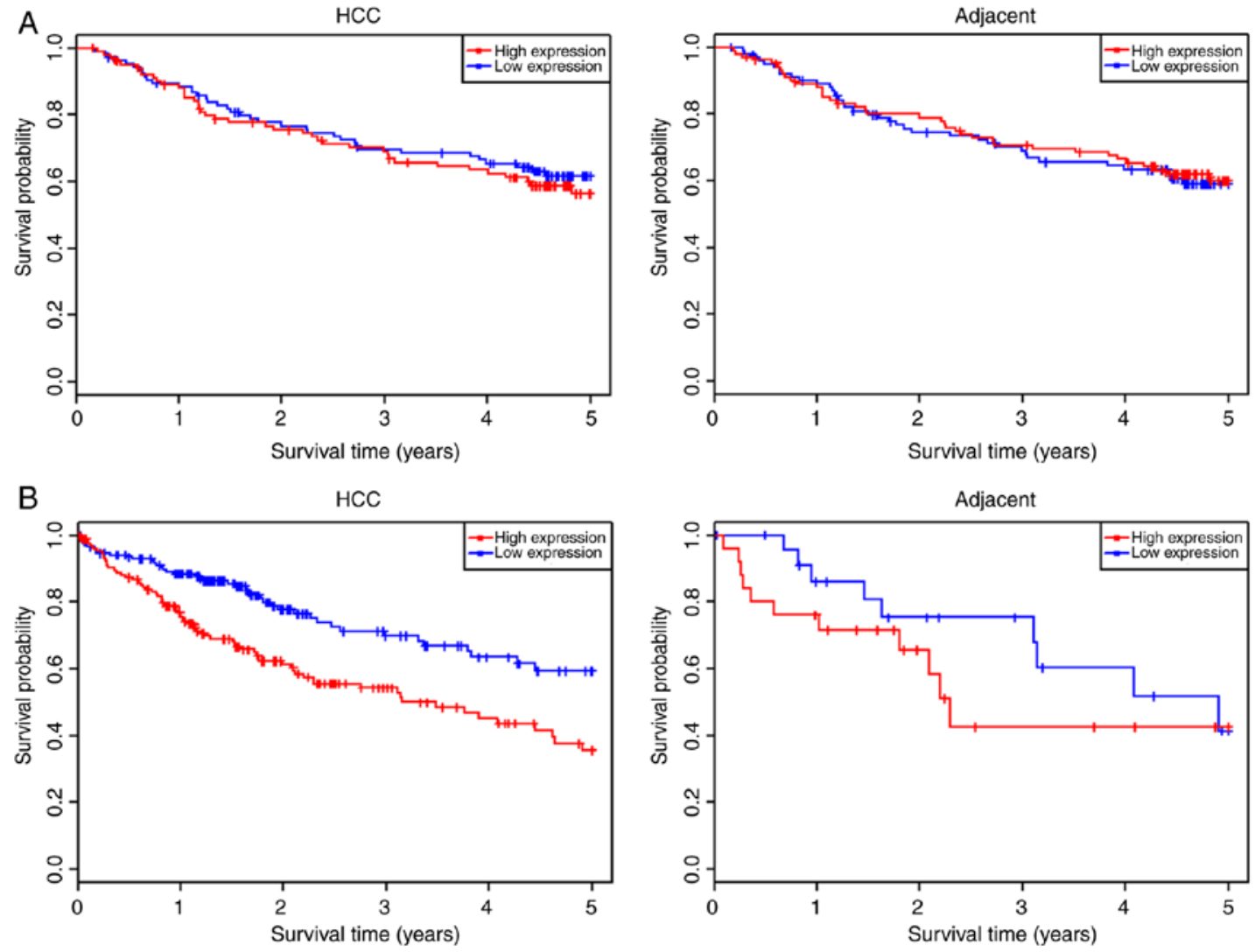

Figure 4. Effect of BCAT1 in survival of HCC. (A) Survival of patients with no significant difference of BCAT1 expression between HCC tissues and adjacent tissues. (B) Survival of patients with significant difference of BCAT1 expression between HCC tissues and adjacent tissues. BCAT1, branched chain amino acid transaminase $1 ; \mathrm{HCC}$, hepatocellular carcinoma.

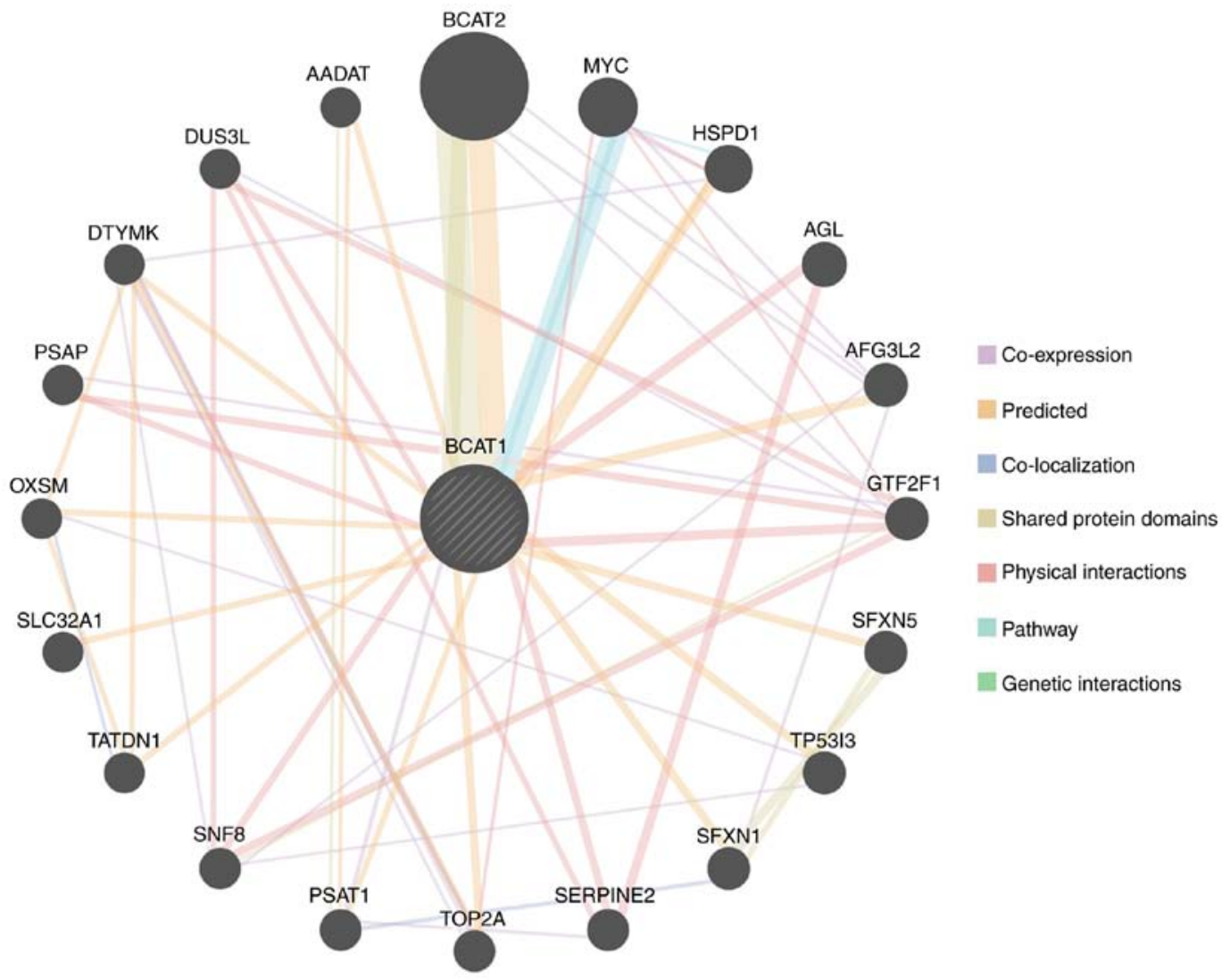

Figure 5. Biological interaction network of BCAT1. Different colors represent diverse bioinformatics methods. BCAT1, branched chain amino acid transaminase 1 . 
A

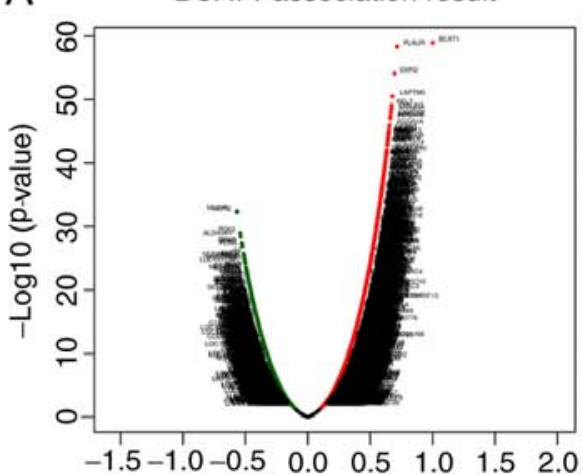

B Positively correlated genes

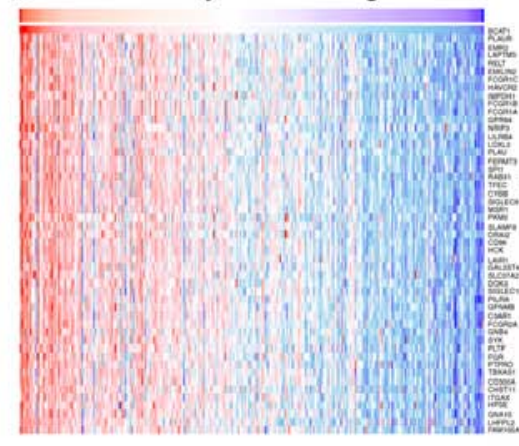

C Negatively correlated genes

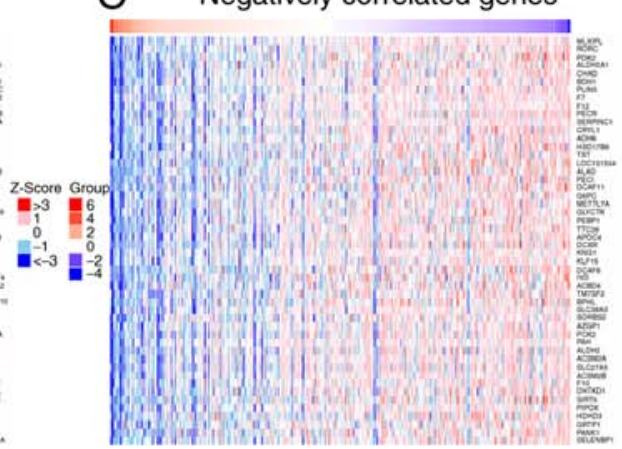

Pearson correlation coefficient (pearson test)

Figure 6. Differentially expressed genes in correlation with $B C A T 1$. (A) Correlations between $B C A T 1$ and differentially express of genes in HCC were analyzed by Pearson test. (B) Heat map of positively correlated genes with $B C A T 1$ in HCC. (C) Heat map of negatively correlated genes with $B C A T 1$ in HCC. Red indicates positive and blue indicates negative. BCAT1, branched chain amino acid transaminase 1; HCC, hepatocellular carcinoma.
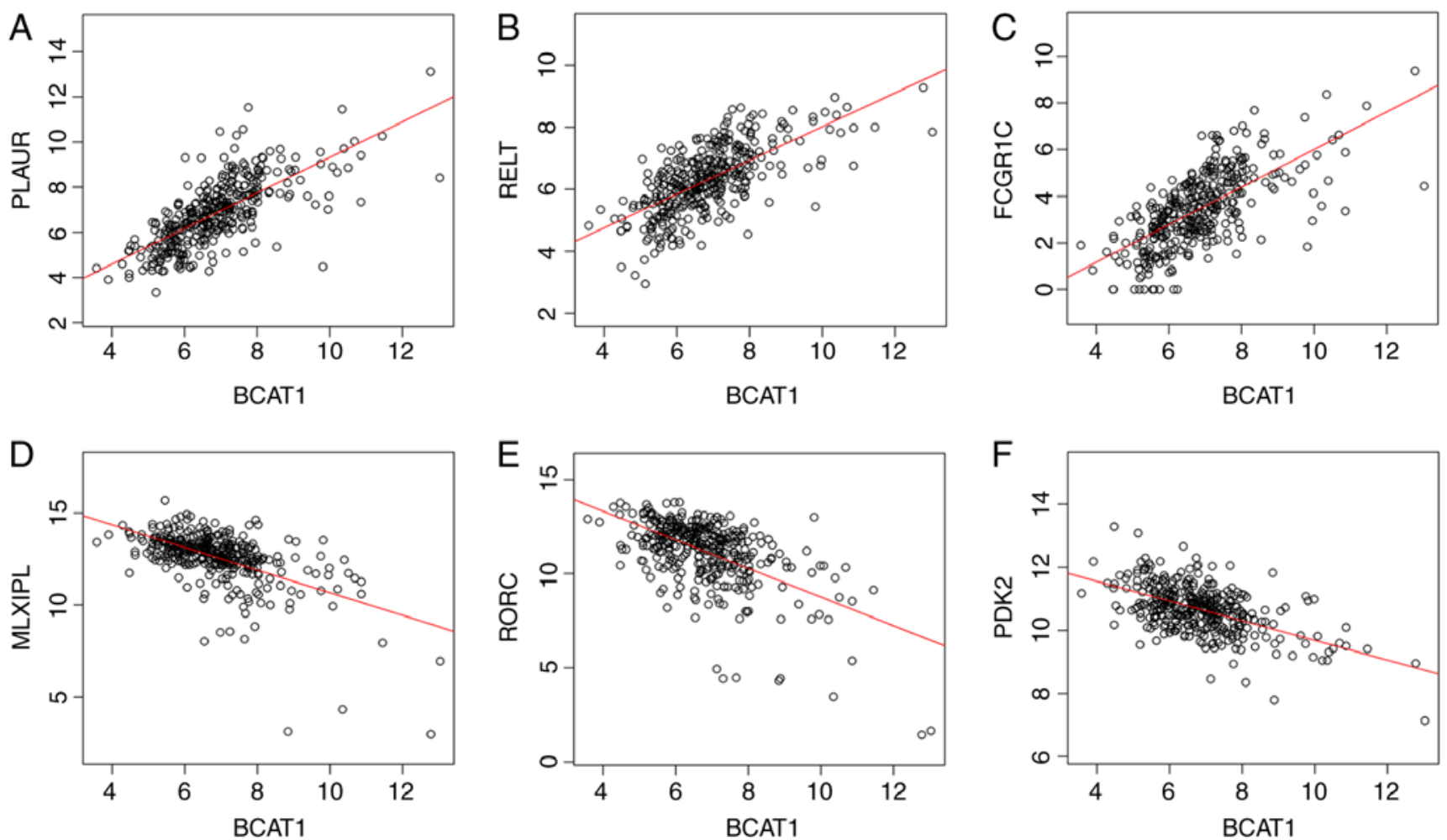

Figure 7. Gene expression correlation analysis of $B C A T 1$ and other genes. Pearson correlation of $B C A T 1$ expression with (A) PLAUR expression, (B) RELT expression, (C) FCGR1C expression, (D) MLXIPL expression, (E) RORC expression and (F) PDK2 expression. BCAT1, branched chain amino acid transaminase 1; FCGR1C, Fc fragment of IgG receptor Ic; PDK2, pyruvate dehydrogenase kinase 2; PLAUR, plasminogen activator, urokinase receptor; RORC, RAR related orphan receptor C; MLXIPL, MLX interacting protein like.

BCAT1 and TP53 was previously unacknowledged. TP53I3 is induced by the tumor suppressor p53 and is involved in p53-mediated apoptosis (62). The present study predicted that $B C A T 1$ may be involved in p53 signaling via its interaction with TP53I3, suggesting that BCAT1 serves a more important role in HCC than was originally hypothesized. In addition to TP53I3, BCAT1 was predicted to interact with AADAT, TOP2A and DTYMK, which are involved in tryptophan and lysine metabolism, DNA topology state regulation and pyrimidine metabolism, respectively. These predictions indicated that $B C A T 1$ may participate in a large number of biological functions in $\mathrm{HCC}$ via its potential interaction with (or regulation of) critical factors. In HCC, $B C A T 1$ was predicted to associate with a number of critical genes including $R E L T, P D K 2$ and $R O R C$, which regulate T-cell responses, apoptosis, the tricarboxylic acid cycle and inflammation (63-65).

However, there were limitations to the present study. First, only 371 tumor cases and 50 normal controls were employed for analysis, and a larger number of patients is required to verify the present findings. In addition, few patients at cancer stage 4 , between 81 and 100 years of age and with extreme 


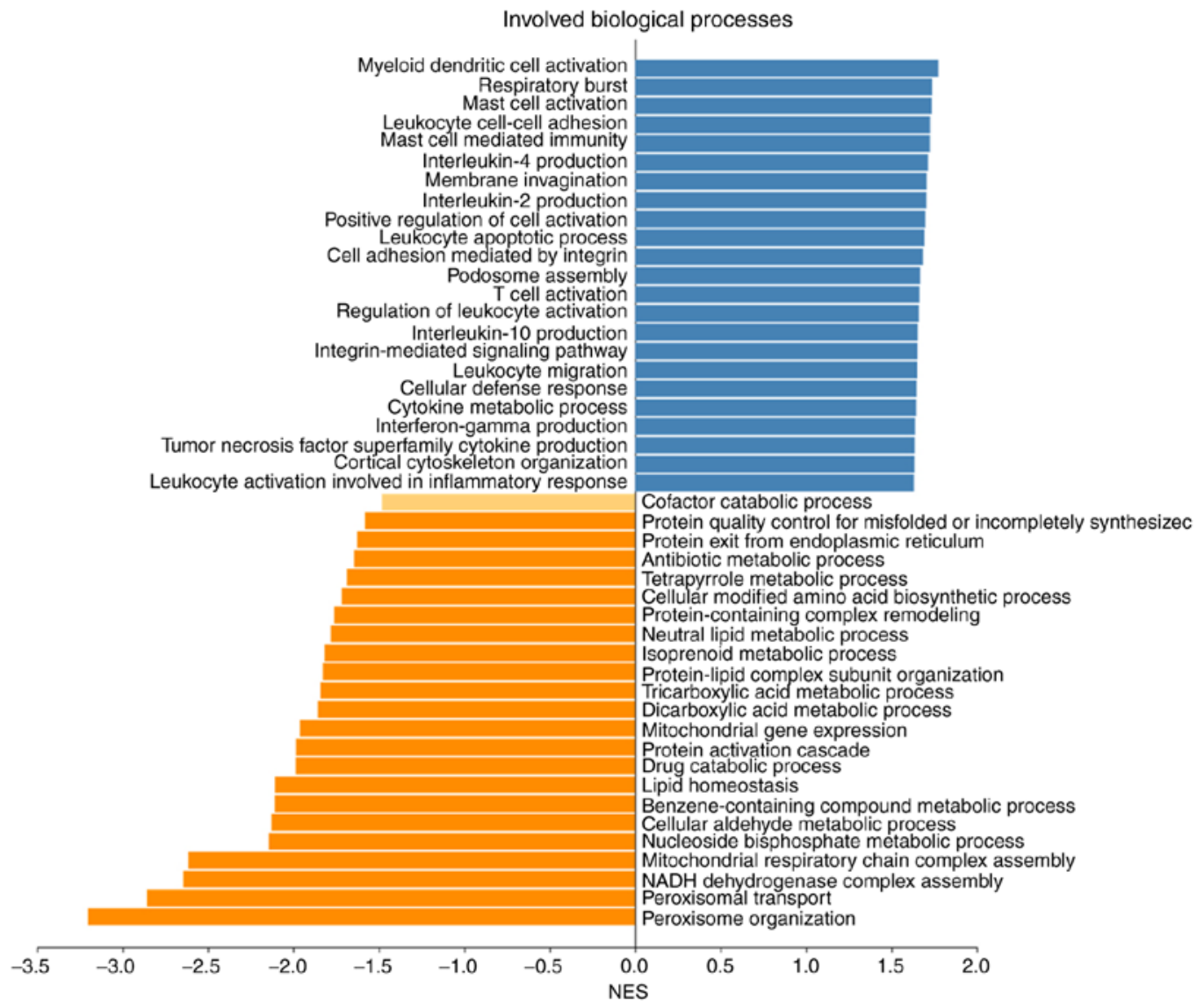

B

Involved molecular functions

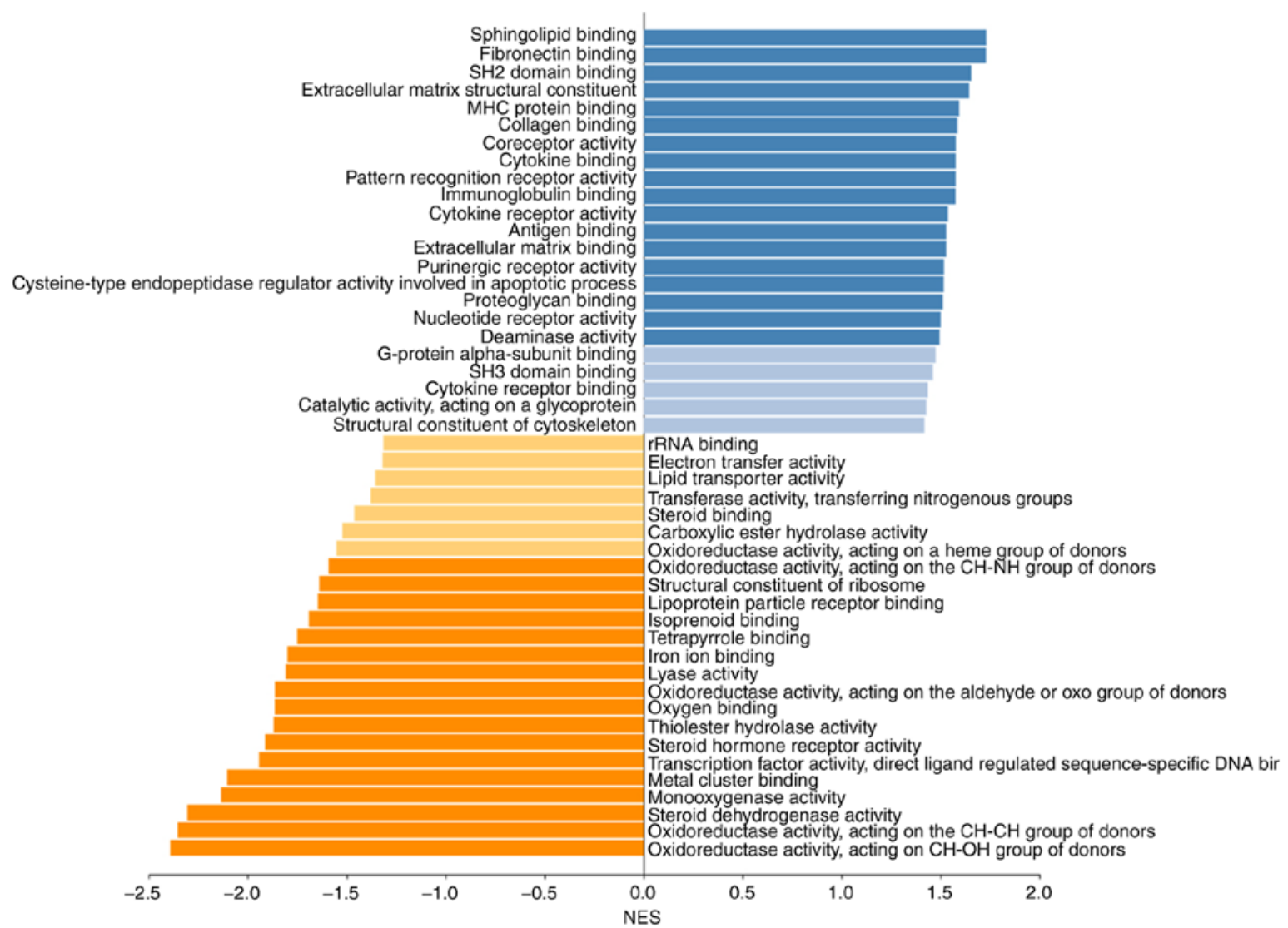

Figure 8. Enriched Gene Ontology annotations of BCAT1 correlated genes in hepatocellular carcinoma. (A) Biological process analysis. (B) Molecular function analysis. Dark blue and orange indicate FDR $\leq 0.05$, light blue and orange indicate FDR $>0.05$. BCAT1, branched chain amino acid transaminase 1; FDR, false discovery rate. 
obesity were included, which may suggest that a large number of HCC patients die prior to diagnosis. Secondly, only bioinformatics analysis results were displayed in this study. Further verification experiments, such as PCR experiments, western blot assays and co-immunoprecipitation, are necessary to verify the present results. Another limitation is that transcriptome sequencing cannot directly provide information on protein activity, which can only be verified by follow-up studies.

In conclusion, the present study provides bioinformatics evidence of the significance of BCATl in hepatocarcinogenesis and its potential as an early detection marker. The results indicated that the function and effects of $B C A T 1$ in HCC are multifaceted, and that $B C A T 1$ may be associated with immune activation, metabolic pathways and the cell cycle. Furthermore, a $B C A T 1$ miRNA-target network was analyzed using TargetScan, demonstrating that thousands of miRNAs were predicted to interact with BCATl (data not shown) and providing a clear direction for future studies. More importantly, analysis results of the present study of this well-known gene indicated that the interactions between $B C A T 1$ and numerous other critical proteins were uncharted. This will help us to determine the future research direction and draw a complete molecular network of $B C A T 1$ participating in HCC. Also, the present study provided researchers, especially young researchers who lack experimental and bioinformatics analysis platforms, with guidance on finding directions for functional genome research.

\section{Acknowledgements}

Not applicable.

\section{Funding}

The present study was supported in part by the National Natural Science Foundation of China (grant no. 81773148), the National Key Sci-Tech Special Project of China (grant no. 2018ZX10302207), the Natural Science Foundation of Guangxi (grant no. 2018GXNSFDA138001), the Basic Ability Enhancement Program for Young and Middle-aged Teachers of Guilin Medical University (grant no. 2018glmcy073) and the Basic Ability Enhancement Program for Young and Middle-aged Teachers of Guangxi (grant no. 2019KY0553).

\section{Availability of data and materials}

The datasets generated and/or analyzed during the current study are available in the TCGA repository, https://portal.gdc.cancer. gov/; the GEO repository (https://www.ncbi.nlm.nih.gov/geo/ query/acc.cgi?acc=GSE25097, https://www.ncbi.nlm.nih.gov/ geo/query/acc.cgi?acc=GSE22058, https://www.ncbi.nlm.nih. gov/geo/query/acc.cgi?acc=GSE36376, https://www.ncbi.nlm.nih. gov/geo/query/acc.cgi?acc=GSE14520, https://www.ncbi.nlm.nih. gov/geo/query/acc.cgi?acc=GSE10143, https://www.ncbi.nlm.nih. gov/geo/query/acc.cgi?acc=GSE9843, https://www.ncbi.nlm.nih. gov/geo/query/acc.cgi?acc=GSE19977, https://www.ncbi.nlm.nih. gov/geo/query/acc.cgi?acc=GSE46444, https://www.ncbi.nlm. nih.gov/geo/query/acc.cgi?acc=GSE54236, https://www.ncbi. nlm.nih.gov/geo/query/acc.cgi?acc=GSE63898, https://www.
ncbi.nlm.nih.gov/geo/query/acc.cgi?acc=GSE43619, https://www. ncbi.nlm.nih.gov/geo/query/acc.cgi?acc=GSE64041, https:// www.ncbi.nlm.nih.gov/geo/query/acc.cgi?acc=GSE76427); and the ICGC repository, https://dcc.icgc.org/projects/LIRI-JP.

\section{Authors' contribution}

RZY and WJL conceived, designed the research and drafted the manuscript; HFZ and WTX were responsible for the acquisition and analysis of data; HFZ and MJL analyzed and interpreted the data; RZY revised the manuscript for important intellectual content. All authors read and approved the manuscript and agree to be accountable for all aspects of the research in ensuring that the accuracy or integrity of any part of the work are appropriately investigated and resolved.

\section{Ethics approval and consent to participate}

Not applicable.

\section{Patient consent for publication}

Not applicable.

\section{Competing interests}

The authors declare that they have no competing interests.

\section{References}

1. Bray F, Ferlay J, Soerjomataram I, Siegel RL, Torre LA and Jemal A: Global cancer statistics 2018: GLOBOCAN estimates of incidence and mortality worldwide for 36 cancers in 185 countries. CA Cancer J Clin 68: 394-424, 2018.

2. Villanueva A: Hepatocellular carcinoma. N Eng J Med 380: 1450-1462, 2019.

3. Dhanasekaran R, Limaye A and Cabrera R: Hepatocellular carcinoma: Current trends in worldwide epidemiology, risk factors, diagnosis, and therapeutics. Hepat Med 4: 19-37, 2012.

4. Thewes V, Simon R, Hlevnjak M, Schlotter M, Schroeter P, Schmidt K, Wu Y, Anzeneder T, Wang W, Windisch P, et al: The branched-chain amino acid transaminase 1 sustains growth of antiestrogen-resistant and ERalpha-negative breast cancer. Oncogene 36: 4124-4134, 2017.

5. Wang ZQ, Faddaoui A, Bachvarova M, Plante M, Gregoire J, Renaud MC, Sebastianelli A, Guillemette C, Gobeil S, Macdonald E, et al: BCAT1 expression associates with ovarian cancer progression: Possible implications in altered disease metabolism. Oncotarget 6: 31522-31543, 2015.

6. Xu Y, Yu W, Yang T, Zhang M, Liang C, Cai X and Shao Q: Overexpression of BCAT1 is a prognostic marker in gastric cancer. Hum Pathol 75: 41-46, 2018.

7. Mayers JR, Torrence ME, Danai LV, Papagiannakopoulos T, Davidson SM, Bauer MR, Lau AN, Ji BW, Dixit PD, Hosios AM, et al: Tissue of origin dictates branched-chain amino acid metabolism in mutant Kras-driven cancers. Science 353: 1161-1165, 2016.

8. Zheng YH, Hu WJ, Chen BC, Grahn TH, Zhao YR, Bao HL, Zhu YF and Zhang QY: BCAT1, a key prognostic predictor of hepatocellular carcinoma, promotes cell proliferation and induces chemoresistance to cisplatin. Liver Int 36: 1836-1847, 2016.

9. Xu M, Liu Q, Jia Y, Tu K, Yao Y, Liu Q and Guo C: BCAT1 promotes tumor cell migration and invasion in hepatocellular carcinoma. Oncol Lett 12: 2648-2656, 2016.

10. Tonjes M, Barbus S, Park YJ, Wang W, Schlotter M, Lindroth AM, Pleier SV, Bai AHC, Karra D, Piro RM, et al: BCAT1 promotes cell proliferation through amino acid catabolism in gliomas carrying wild-type IDH1. Nat Med 19: 901-908, 2013. 
11. Zhou W, Feng X, Ren C, Jiang X, Liu W, Huang W, Liu Z, Li Z, Zeng L, Wang L, et al: Over-expression of BCAT1, a c-Myc target gene, induces cell proliferation, migration and invasion in nasopharyngeal carcinoma. Mol Cancer 12: 53, 2013.

12. Raffel S, Falcone M, Kneisel N, Hansson J, Wang W, Lutz C, Bullinger L, Poschet G, Nonnenmacher Y, Barnert A, et al: BCAT1 restricts $\alpha \mathrm{KG}$ levels in AML stem cells leading to IDHmut-like DNA hypermethylation. Nature 551: 384-388, 2017.

13. Papathanassiu AE, Ko JH, Imprialou M, Bagnati M, Srivastava PK, Vu HA, Cucchi D, McAdoo SP, Ananieva EA, Mauro C and Behmoaras J: BCAT1 controls metabolic reprogramming in activated human macrophages and is associated with inflammatory diseases. Nat Commun 8: 16040, 2017.

14. Wang HG, Xie R, Shen P, Huang XD, Ji GZ and Yang XZ: BCAT1 expression in hepatocellular carcinoma. Clin Res Hepatol Gastroenterol 40: e55-e56, 2016.

15. Chandrashekar DS, Bashel B, Balasubramanya SAH, Creighton CJ, Ponce-Rodriguez I, Chakravarthi BVSK and Varambally S: UALCAN: A portal for facilitating tumor subgroup gene expression and survival analyses. Neoplasia 19 : 649-658, 2017.

16. Warde-Farley D, Donaldson SL, Comes O, Zuberi K, Badrawi R, Chao P, Franz M, Grouios C, Kazi F, Lopes CT, et al: The GeneMANIA prediction server: Biological network integration for gene prioritization and predicting gene function. Nucleic Acids Res 38: W214-W220, 2010.

17. Vasaikar SV, Straub P, Wang J and Zhang B: LinkedOmics: Analyzing multi-omics data within and across 32 cancer types. Nucleic Acids Res 46: D956-D963, 2018.

18. Wang J, Vasaikar S, Shi Z, Zhang B and Greer M: WebGestalt 2017: A more comprehensive, powerful, flexible and interactive gene set enrichment analysis toolkit. Nucleic Acids Res 45: W130-W137, 2017

19. Lian Q, Wang S, Zhang G, Wang D, Luo G, Tang J, Chen L and Gu J: HCCDB: A database of hepatocellular carcinoma expression atlas. Genomics Proteomics Bioinformatics 16: 269-275, 2018

20. Tung EK, Mak CK, Fatima S, Lo RC, Zhang C, Dai H, Poon RT, Yuen MF, Lai CL, Li JJ, et al: Clinicopathological and prognostic significance of serum and tissue Dickkopf-1 levels in human hepatocellular carcinoma. Liver Int 31: 1494-1504, 2011

21. Lamb JR, Zhang C, Xie T, Wang K, Zhang B, Hao K, Chudin E, Fraser HB, Millstein J, Ferguson M, et al: Predictive genes in adjacent normal tissue are preferentially altered by sCNV during tumorigenesis in liver cancer and may rate limiting. PloS One 6: e20090, 2011.

22. Sung WK, Zheng H, Li S, Chen R, Liu X, Li Y, Lee NP, Ariyaratne PN, Tennakoon C, Mulawadi FH, et al: Genome-wide survey of recurrent $\mathrm{HBV}$ integration in hepatocellular carcinoma. Nat Genet 44: 765-769, 2012.

23. Wong KF, Liu AM, Hong $\mathrm{W}, \mathrm{Xu} \mathrm{Z}$ and Luk JM: Integrin $\alpha 2 \beta 1$ inhibits MST1 kinase phosphorylation and activates Yes-associated protein oncogenic signaling in hepatocellular carcinoma. Oncotarget 7: 77683-77695, 2016.

24. Liu AM, Yao TJ, Wang W, Wong KF, Lee NP, Fan ST, Poon RT, Gao C and Luk JM: Circulating miR-15b and miR-130b in serum as potential markers for detecting hepatocellular carcinoma: a retrospective cohort study. BMJ Open 2: e000825, 2012.

25. Burchard J, Zhang C, Liu AM, Poon RT, Lee NP, Wong KF, Sham PC,Lam BY,Ferguson MD, Tokiwa G, et al: microRNA-122 as a regulator of mitochondrial metabolic gene network in hepatocellular carcinoma. Mol Sys Biol 6: 402,2010.

26. Lim HY, Sohn I, Deng S, Lee J, Jung SH, Mao M, Wang K, Shi S Joh JW, et al: Prediction of disease-free survival in hepatocellular carcinoma by gene expression profiling. Ann Surg Oncol 20: 3747-3753, 2013

27. Roessler S, Jia HL, Budhu A, Forgues M, Ye QH, Lee JS, Thorgeirsson SS, Sun Z, Tang ZY, Qin LX and Wang XW: A unique metastasis gene signature enables prediction of tumor relapse in early-stage hepatocellular carcinoma patients. Cancer Res 70: 10202-10212, 2010.

28. Roessler S, Long EL, Budhu A, Chen Y, Zhao X, Ji J, Walker R, Jia HL, Ye QH, Qin LX, et al: Integrative genomic identification of genes on $8 \mathrm{p}$ associated with hepatocellular carcinoma progression and patient survival. Gastroenterology 142: 957-966.e912, 2012.

29. Zhao X, Parpart S, Takai A, Roessler S, Budhu A, Yu Z, Blank M, Zhang YE, Jia HL, Ye QH, et al: Integrative genomics identifies YY1AP1 as an oncogenic driver in $\operatorname{EpCAM}(+) \operatorname{AFP}(+)$ hepatocellular carcinoma. Oncogene 34: 5095-5104, 2015.
30. Wang Y, Gao B, Tan PY, Handoko YA, Sekar K, Deovasogamani A, Seshachalam VP, OuYang HY, Shi M, Xie C, et al: Genome-wide CRISPR knockout screens identify NCAPG as an essential oncogene for hepatocellular carcinoma tumor growth. FASEB J 33: 8759-8770, 2019.

31. Hoshida Y, Villanueva A, Kobayashi M, Peix J, Chaing DY, Camargo A, Gupta S, Moore J, Wrobel MJ, Lerner J, et al: Gene expression in fixed tissues and outcome in hepatocellular carcinoma. N Engl J Med 359: 1995-2004, 2008.

32. Chiang DY, Villanueva A, Hoshida Y, Peix J, Newell P, Minguez B, LeBlanc AC, Donovan DJ, Thung SN, Solé M, et al: Focal gains of VEGFA and molecular classification of hepatocellular carcinoma. Cancer Res 68: 6779-6788, 2008.

33. Villanueva A, Hoshida Y, Battiston C, Tovar V, Sia D, Alsinet C, Cornella H, Liberzon A, Kobayashu M, Kumada H, et al: Combining clinical, pathology, and gene expression data to predict recurrence of hepatocellular carcinoma. Gastroenterology 140: 1501-1512.e1502, 2011

34. Toffanin S, Hoshida Y, Lachenmayer A, Villanueva A, Cabellos L, Minguez B, Savic R, Ward SC, Thung S, Chiang DY, et al: MicroRNA-based classification of hepatocellular carcinoma and oncogenic role of miR-517a. Gastroenterology 140: 16181628.e1616, 2011.

35. Kishikawa T, Otsuka M, Tan PS, Ohno M, Sun X, Yoshikawa T, Shibata C, Takata A, Kojima K, Takehana K, et al: Decreased miR122 in hepatocellular carcinoma leads to chemoresistance with increased arginine. Oncotarget 6: 8339-8352, 2015.

36. Kojima K, April C, Canasto-Chibuque C, Chen X, Deshmukh M, Venkatesh A, Tan PS, Kobayashi M, Kumada H, Fan JB and Hoshida Y: Transcriptome profiling of archived sectioned formalin-fixed paraffin-embedded (AS-FFPE) tissue for disease classification. PloS One 9: e86961, 2014.

37. Villa E, Critelli R, Lei B, Marzocchi G, Cammà C, Giannelli G, Cabibbo G, Enea M, Colopi S, Caporali C, et al: Neoangiogenesis-related genes are hallmarks of fast-growing hepatocellular carcinomas and worst survival. Results from a prospective study. Gut 65: 861-869, 2016

38. Zubiete-Franco I, Garcia-Rodriguez JL, Lopitz-Otsoa F, Serrano Macia M, Simon J, Fernàndez-Tussy $\mathrm{P}$, Barbier-Torres L, Fernàndez-Ramos D, Gutiérrez-de-Juan V, López de Davalillo S, et al: SUMOylation regulates LKB1 localization and its oncogenic activity in liver cancer. EBioMedicine 40: 406-421, 2019.

39. Villanueva A, Portela A, Sayols S, Battiston C, Hoshida Y, Méndez-González J, Imbaud S, Letouzé E, Hernandez-Gea V, Corenlla $\mathrm{H}$, et al; HEPTROMIC Consortium: DNA methylation-based prognosis and epidrivers in hepatocellular carcinoma. Hepatology 61: 1945-1956, 2015.

40. Dong B, Lee JS, Park YY, Yang F, Xu G, Huang W, Finegold J and Moore DD: Activating CAR and $\beta$-catenin induces uncontrolled liver growth and tumorigenesis. Nat Commun 6: $5944,2015$.

41. Makowska Z, Boldanova T, Adametz D, Quagliata L, Vogt JE, Dill MT, Matter MS, Roth V, Terracciano L and Heim MH: Gene expression analysis of biopsy samples reveals critical limitations of transcriptome-based molecular classifications of hepatocellular carcinoma. J Pathol Clin Res 2: 80-92, 2016.

42. Grinchuk OV, Yenamandra SP, Iyer R, Singh M, Lee HK, Lim KH, Chow PK and Kuznetsov VA: Tumor-adjacent tissue co-expression profile analysis reveals pro-oncogenic ribosomal gene signature for prognosis of resectable hepatocellular carcinoma. Mol Oncol 12: 89-113, 2018.

43. Liao MJ, Yang XM, Yao RZ and Shi N: BCAT1 overexpression associates with clinical progression and poor prognosis in patients with hepatocellular carcinoma. Int $\mathbf{J}$ Clin Exp Med 12: 4202-4209, 2019.

44. Lyssiotis CA and Kimmelman AC: Metabolic interactions in the tumor microenvironment. Trends Cell Biol 27: 863-875, 2017.

45. Reina-Campos M, Moscat J and Diaz-Meco M: Metabolism shapes the tumor microenvironment. Curr Opin Cell Biol 48: 47-53, 2017.

46. Ananieva EA and Wilkinson AC: Branched-chain amino acid metabolism in cancer. Curr Opin Clin Nutr Metab Care 21: 64-70, 2018

47. Dey P, Baddour J, Muller F, Wu CC, Wang H, Liao WT, Lan Z, Chen A, Gutschner T, Kang Y, et al: Genomic deletion of malic enzyme 2 confers collateral lethality in pancreatic cancer. Nature 542: 119-123, 2017. 
48. Symonds EL, Pedersen SK, Baker RT, Murray DH, Gaur S, Cole SR, Gopalsamy G, Mangira D, LaPointe LC and Young GP: A blood test for methylated BCAT1 and IKZF1 vs. a fecal immunochemical test for detection of colorectal neoplasia. Clin Transl Gastroenterol 7: e137, 2016.

49. Li Q, Pan X, Zhu D, Deng Z, Jiang R and Wang X: Circular RNA MAT2B promotes glycolysis and malignancy of hepatocellular carcinoma via the miR-338-3p/PKM2 axis under hypoxic stress. Hepatology: Apr 20, 2019 (Epub ahead of print).

50. Itzel T, Spang R, Maass T, Munker S, Roessler S, Ebert MP Schlitt HJ, Herr W, Evert M and Teufel A: Random gene sets in predicting survival of patients with hepatocellular carcinoma. J Mol Med (Berl) 97: 2019.

51. Yu H, Wang $\mathrm{H}, \mathrm{Xu} \mathrm{HR}$, Zhang $\mathrm{YC}, \mathrm{Yu} \mathrm{XB}, \mathrm{Wu} \mathrm{MC}$, Jin GZ and Cong WM: Overexpression of MTHFD1 in hepatocellular carcinoma predicts poorer survival and recurrence. Future Oncol 15 1771-1780, 2019

52. Jiang H, Zhang X, Tao Y, Shan L, Jiang Q, Yu Y, Cai F and Ma L: Prognostic and clinicopathologic significance of SIRT1 expression in hepatocellular carcinoma. Oncotarget 8: 52357-52365, 2016.

53. Hanahan D and Weinberg RA: Hallmarks of cancer: The next generation. Cell 144: 646-674, 2011.

54. Zhang L and Han J: Branched-chain amino acid transaminase 1 (BCAT1) promotes the growth of breast cancer cells through improving mTOR-mediated mitochondrial biogenesis and function. Biochem Biophys Res Commun 486: 224-231, 2017.

55. Eden $A$ and Benvenisty N: Involvement of branched-chain amino acid aminotransferase (Bcat1/Eca39) in apoptosis. FEBS Lett 457: 255-261, 1999.

56. Ji D, Jiang C, Zhang L, Liang N, Jiang T, Yang B and Liang H: LncRNA CRNDE promotes hepatocellular carcinoma cell proliferation, invasion, and migration through regulating miR-203/ BCAT1 axis. J Cell Physiol 234: 6548-6560, 2019.

57. Qi LN, Xiang BD, Wu FX, Ye JZ, Zhong JH, Wang YY, Chen YY, Chen ZS, Ma L, Chen J, et al: Circulating tumor cells undergoing EMT provide a metric for diagnosis and prognosis of patients with hepatocellular carcinoma. Cancer Res 78: 4731-4744, 2018.
58. Cho HR, Jeon H,Park CK, Park SH, Kang KM and Choi SH: BCAT1 is a new MR imaging-related biomarker for prognosis prediction in IDH1-wildtype glioblastoma patients. Sci Rep 7: 17740, 2017.

59. Li H, Ye D, Xie W, Hua F, Yang Y, Wu J, Gu A, Ren Y and Mao K: Defect of branched-chain amino acid metabolism promotes the development of Alzheimer's disease by targeting the mTOR signaling. Biosci Rep 38: pii: BSR20180127, 2018.

60. Jour G, Vasudevaraja V, Prieto VG, Snuderl M, Torres-Cabala CA, Al-Rohil R, Sulman EP, Ballester LY and Aung PP: BCAT1 and miR-2504: Novel methylome signature distinguishes spindle/desmoplastic melanoma from superficial malignant peripheral nerve sheath tumor. Mod Pathol 32: 338-345, 2019.

61. Honda M, Takehana K, Sakai A, Tagata Y, Shirasaki T, Nishitani S, Muramatsu T, Yamashita T, Nakamoto Y, Mizukoshi E, et al: Malnutrition impairs interferon signaling through mTOR and FoxO pathways in patients with chronic hepatitis C. Gastroenterology 141: 128-140, 2011.

62. Chu Y, Li D, Zhang H, Ding J, Xu P, Qiu X and Zhang H: PIG3 suppresses gastric cancer proliferation by regulating p53-mediated apoptosis. J Biol Regul Homeost Agents 32: 1185-1189, 2018.

63. Moua P, Checketts M, Xu LG, Shu HB, Reyland ME and Cusick JK: RELT family members activate p38 and induce apoptosis by a mechanism distinct from TNFR1. Biochem Biophys Res Commun 491: 25-32, 2017.

64. Choi BK, Kim SH, Kim YH, Lee DG, Oh HS, Han C, Kim YI, Jeon $\mathrm{Y}$, Lee $\mathrm{H}$ and Kwon BS: RELT negatively regulates the early phase of the T-cell response in mice. Eur J Immunol 48: $1739-1749,2018$

65. Go Y, Jeong JY, Jeoung NH, Jeon JH, Park BY, Kang HJ, Ha CM, Choi YK, Lee SJ, Ham HJ, et al: Inhibition of pyruvate dehydrogenase kinase 2 protects against hepatic steatosis through modulation of tricarboxylic acid cycle anaplerosis and ketogenesis. Diabetes 65: 2876-2887, 2016.

This work is licensed under a Creative Commons

Attribution-NonCommercial-NoDerivatives 4.0 International (CC BY-NC-ND 4.0) License. 